\title{
ON SOME MERCERIAN THEOREMS IN SUMMABILITY
}

\section{R. PARAMESWARAN}

1. Introduction. Mercer's Theorem [10;4, Theorem 51] and various extensions of it have been treated in many recent papers $[1 ; \mathbf{3}$; $7 ; 8$ ] etc. It is the object of this paper to use functional analysis to prove Mercerian Theorems for ordinary and absolute summability; we prove also a few results on absolute summability that may have some independent interest. Functional analysis treatment of Mercerian theorems for ordinary summability has also been given by Sirkov $[13] .^{1}$

2. Some notations and lemmas. We note the following definitions and lemmas, some of which are quite well known. We shall use the symbols $\left(c_{0}\right),(c)$ and $(m)$ to denote respectively the set of all sequences converging to zero, the set of all convergent sequences, and the set of all bounded sequences. The sequence-to-sequence transformation given by the equations

$$
y_{n}=\sum_{k=0}^{\infty} a_{n k} x_{k}, \quad(n=0,1,2, \cdots)
$$

will be written as a matrix equation $y=A x$ where $y$ and $x$ are column vectors, $y=\left\{y_{n}\right\}$ and $x=\left\{x_{n}\right\}$. Let $(\mathfrak{M})$ and $(\mathfrak{R})$ be given sets of sequences. Then $\Gamma(\mathfrak{M}, \mathfrak{R})$ will denote the set of matrices $A$ such that $x \in(\mathfrak{M})$ implies $A x \in(\mathfrak{R})$. As we shall see, it will be convenient to work with this notation.

Lemma 1 (Hille [5, p. 92]). If $\Gamma$ is any complex Banach algebra with unit element $I$, then for every element $A \in \Gamma$ the element $I+q A$ where $q$ is any complex number such that $|q| \cdot\|A\|<1$, has an inverse in $\Gamma$.

Lemma 2. The sets of matrices $\Gamma\left(c_{0}, c_{0}\right), \Gamma(c, c)$ and $\Gamma(m, m)$ are all complex Banach algebras, with the norm in each case being defined by: $\|A\|=$ l.u.b. $0 \leqq n<\infty<\sum_{k=0}^{\infty}\left|a_{n k}\right|$.

A proof for $\Gamma(c, c)$ is found in the author's paper [11]; the other cases are similarly proved.

Lemma 3. (Zeller [14]). Let $A \in \Gamma(\mathfrak{M}, \mathfrak{M}),(\mathfrak{M})=\left(c_{0}\right)$ or $(c)$. If $A x \in(\mathfrak{M})$ implies $x \in(m)$, then $x \in(\mathfrak{M})$.

Received by the editors August 23, 1956 and, in revised form, March 4, 1957.

1 The author is thankful to the referee for this and other valuable remarks. 
Lemma 4. Let $A \in \Gamma(\mathfrak{M}, \mathfrak{M}),(\mathfrak{M})=\left(c_{0}\right)$ or $(c)$. If the matrix $B$ is such that $A B=B A=I$ and $B \in \Gamma(m, m)$ then $B \in \Gamma(\mathfrak{M}, \mathfrak{M})$.

The case $(\mathfrak{M})=(c)$ is proved elsewhere [12]; the other case is proved similarly.

3. Some Mercerian theorems. We now give two theorems, the first of which includes, and slightly generalizes, Theorems $1,2,3$ and 4 of Love [8], and is itself included in the second theorem the results of which were first proved by Agnew (see $[1 ; 2]$ ).

Theorem 1. Let $A \in \Gamma(\mathfrak{M}, \mathfrak{M})$ where $(\mathfrak{M})=\left(c_{0}\right),(c)$ or $(m)$. Then the conditions $(I+q A) x \in(\mathfrak{M})$,

$$
|q| \cdot \limsup _{n \rightarrow \infty} \sum_{k=0}^{\infty}\left|a_{n k}\right|<1
$$

imply that $x \in(\mathfrak{M})$ provided that either (i) $x \in(m)$ or (ii) $A$ is a lowersemimatrix (i.e., $a_{n k}=0$ for $k>n$ ).

Theorem 2. Let $A \in \Gamma(\mathfrak{M}, \mathfrak{M}),(\mathfrak{M})=\left(c_{0}\right)$ or $(c)$ or $(m)$ and let

$$
\liminf _{n \rightarrow \infty}\left\{\left|a_{n n}\right|-\sum_{k \neq n}\left|a_{n k}\right|\right\}>\lambda>0
$$

then $A x \in(\mathfrak{M})$ implies that $x \in(\mathfrak{M})$, provided that either (i) $x \in(m)$ or (ii) $A$ is a lower-semimatrix.

Proof. We may assume without loss of generality that $\left|a_{n n}\right|$ $-\sum_{k \neq n}\left|a_{n k}\right|>\lambda>0$ for all $n$, for we may alter a finite number of rows of the matrix $A$ without affecting its summability properties. Define the matrix $B$ by $I+B \equiv\left(a_{n k} / a_{n n}\right)$. Since $\|B\|<1$ by $(2)$, we have by Lemmas 1 and 2 that $C=(I+B)^{-1} \in \Gamma(\mathfrak{M}, \mathfrak{M})$. Also $I+B=P A$ where $P=\left(p_{n k}\right)$ is a diagonal matrix with $p_{n n}=1 / a_{n n}$ for all $n$. Since $\|A\| \geqq\left|a_{n n}\right|>\lambda>0$, both $P$ and $P^{-1}$ belong to $\Gamma(m, m)$. Since further $P A C=C P A=I=P^{-1}(P A C) P=A C P$ we see that $A^{-1}=C P \in \Gamma(m, m)$. Therefore, if $A$ is a lower-semimatrix then $A x \in(\mathfrak{M})$ implies $x \in(m)$, and it follows from either of Lemmas 3, 4 that $x \in(\mathfrak{M})$. This proves part (ii) of the theorem.

If $x \in(m)$ and $A x \in(\mathfrak{M})$, then by what has been proved above and Lemma 4 , we see that $A^{-1} \in \Gamma(\mathfrak{M}, \mathfrak{M})$ and part (i) of the theorem is also proved.

4. Absolute summability. Corresponding to the Mercerian theorems for ordinary summability, there are also analogues for absolute summability-where convergence is replaced by absolute convergence. The sequence $x=\left\{x_{n}\right\}$ is said to be absolutely convergent if 
and only if the series $\sum_{n=0}^{\infty}\left(x_{n}-x_{n-1}\right)$ is absolutely convergent $\left(x_{-1}\right.$ is taken to be zero). We shall denote the set of all absolutely convergent sequences by $(|c|)$, and the set of all sequences $x=\left\{x_{n}\right\}$ such that $\sum_{n=0}^{\infty}\left|x_{n}\right|<\infty$, by the symbol $\left(l_{1}\right)$. Theorem 3 below is the analogue of Lemma 2 for absolute summability; we shall prove it by a somewhat indirect method by a number of easy steps, proving a few lemmas which may also be considered to be of some independent interest.

Lemma 5. (See Mears [9]). The matrix $P=\left(p_{n k}\right),(n, k=0,1,2, \cdots)$ belongs to $\Gamma(|c|,|c|)$, that is, $P x \in(|c|)$ for every $x \in(|c|)$ if and only if

$$
\|P\|^{*} \equiv \underset{\substack{\text { l.u.b. } \\ 0 \leqq \infty}}{\infty}\left|\sum_{n=0}^{\infty}\right| \sum_{k=h}^{\infty}\left(p_{n k}-p_{n-1, k}\right) \mid<\infty
$$

where $p_{-1, k}=0$ and

$$
g_{n}=\sum_{k=0}^{\infty} p_{n k} \quad \text { exists for each } n=0,1,2, \cdots
$$

Lemma 6. (See Knopp and Lorentz [6]). The matrix $A=\left(a_{n k}\right)$ belongs to $\Gamma\left(l_{1}, l_{1}\right)$ if and only if

$$
\left\|A^{\prime}\right\| \equiv \underset{0 \leqq k<\infty}{\operatorname{lou} . \mathrm{b} .} \sum_{n=0}^{\infty}\left|a_{n k}\right|<\infty
$$

(or equivalently, if and only if $A^{\prime} \in \Gamma(m, m)$ where $A^{\prime}$ is the transpose of the matrix $A$, so that obviously $\left.\|A\|^{\prime}=\left\|A^{\prime}\right\|\right)$.

Lemma 7. Let $A=\left(a_{n k}\right)$ and let $G=\left(g_{n k}\right)$ be defined by

$$
g_{n k}=a_{0 k}+a_{1 k}+\cdots+a_{n k} \quad(n, k=0,1,2, \cdots) .
$$

Then $A \in \Gamma\left(l_{1}, l_{1}\right)$ if and only if $G \in \Gamma\left(l_{1},|c|\right)$.

The proof is immediate from the equation

$$
\sum_{n=0}^{\infty}\left|\sum_{k=0}^{\infty} a_{n k} u_{k}\right|=\sum_{n=0}^{\infty}\left|\sum_{k=0}^{\infty}\left(g_{n k}-g_{n-1, k}\right) u_{k}\right|,
$$

for the equation (5) is equivalent to the relation

$$
a_{n k}=g_{n k}-g_{n-1, k} \quad(n, k=0,1,2, \cdots) .
$$

Definition. The set of matrices $A \in \Gamma(\mathfrak{M}, \mathfrak{R})$ such that $\lim _{k \rightarrow \infty} a_{n k}$ $=0$ for each $n=0,1,2, \ldots$ will be denoted by $\Gamma_{0}(\mathfrak{M}, \mathfrak{N})$.

Lemma 8. Let the matrices $A$ and $G$ be related as above. Then, $A \in \Gamma_{0}\left(l_{1}, l_{1}\right)$ if and only if $G \in \Gamma_{0}\left(l_{1},|c|\right)$. 
The proof is immediate from equations (5) and (6).

Lемма 9. The sets of matrices $\Gamma\left(l_{1}, l_{1}\right)$ and $\Gamma_{0}\left(l_{1}, l_{1}\right)$ are complex Banach algebras under the norm defined by (4).

This is a consequence of the fact that $\Gamma\left(l_{1}, l_{1}\right)$ and $\Gamma_{0}\left(l_{1}, l_{1}\right)$ are, under the correspondence $A \rightarrow A^{\prime}$, isometric with the Banach algebras (see Lemma 2) $\Gamma(m, m)$ and $\Gamma\left(c_{0}, c_{0}\right)$ respectively.

It is easily verified that the following result is true:

Lemma 10. Let $P=\left(p_{n k}\right) \in \Gamma(|c|,|c|)$ and let

$$
g_{n k}=p_{n k}+p_{n, k+1}+\cdots+\cdots \quad(n, k=0,1,2, \cdots) .
$$

Then $G=\left(g_{n k}\right) \in \Gamma_{0}\left(l_{1},|c|\right)$ and

$$
p_{n k}=g_{n k}-g_{n, k+1} \quad(n, k=0,1,2, \cdots) .
$$

The converse is also true.

REMARK. The statements $A \in \Gamma_{0}\left(l_{1}, l_{1}\right), \quad G \in \Gamma_{0}\left(l_{1},|c|\right)$ and $P \in \Gamma(|c|,|c|)$ are equivalent. For, $s=\left\{s_{n}\right\} \in\left(l_{1}\right)$ if and only if $\sigma=\left\{\sigma_{n}\right\} \in(|c|)$, where $\sigma_{n}=s_{0}+s_{1}+\cdots+s_{n}$. It is easy to verify that if $A \in \Gamma_{0}\left(l_{1}, l_{1}\right)$ and $s \in\left(l_{1}\right)$, then $A s=t=\left\{t_{n}\right\} \in\left(l_{1}\right), G s=\tau \in(|c|)$ where $\tau=\left\{\tau_{n}\right\}, \tau_{n}=t_{0}+t_{1}+\cdots+t_{n}$ and further that $P \sigma=\tau$. The correspondence $A \leftrightarrow G \leftrightarrow P$ is thus the "natural" one.

We have established above one-to-one correspondences between $\Gamma_{0}\left(l_{1}, l_{1}\right)$ and $\Gamma_{0}\left(l_{1},|c|\right)$ on the one hand and between $\Gamma_{0}\left(l_{1},|c|\right)$ and $\Gamma(|c|,|c|)$ on the other. Thus we have the

Lemma 11. The" correspondences $A \leftrightarrow G \leftrightarrow P$ where $A \in \Gamma_{0}\left(l_{1}, l_{1}\right)$, $G \in \Gamma_{0}\left(l_{1},|c|\right)$ and $P \in \Gamma(|c|,|c|)$, as defined above, are one-to-one; and the correspondence $A \leftrightarrow P$ is expressed by either of the equivalent formulae

$$
\begin{aligned}
& p_{n k}=\sum_{i=0}^{\infty}\left(a_{i k}-a_{i, k+1}\right) \quad(n, k=0,1,2, \cdots), \\
& a_{n k}=\sum_{i=k}^{\infty}\left(p_{n i}-p_{n-1, i}\right) \quad(n, k=0,1,2, \cdots) .
\end{aligned}
$$

The equations giving $\left(p_{n k}\right)$ and $\left(a_{n k}\right)$ in terms of each other are verified by simple calculation.

Lemma 12. Let the matrix $G$ be related to $A \in \Gamma\left(l_{1}, l_{1}\right)$ by the equation (5) and let $B \in \Gamma\left(l_{1}, l_{1}\right)$. Then $G B \in \Gamma\left(l_{1},|c|\right)$ and is similarly related to $A B \in \Gamma\left(l_{1}, l_{1}\right)$. 
Proof. The equations (4) and (6) give

$$
\left|g_{n i}\right| \leqq \sum_{k=0}^{\infty}\left|g_{k i}-g_{k-1, i}\right| \leqq\|A\|^{\prime}<\infty ;
$$

also, $\sum_{i=0}^{\infty}\left|b_{i k}\right| \leqq\|B\|^{\prime}<\infty$. Therefore the sums $(G B)_{n k} \equiv \sum_{i=0}^{\infty} g_{n i} b_{i k}$ exist for $n, k=0,1,2, \ldots$; and hence the product $G B$ also exists.

Now, $\quad(A B)_{n k}=\sum_{i=0}^{\infty} a_{n i} b_{i k}=\sum_{i=0}^{\infty}\left(g_{n i}-g_{n-1, i}\right) b_{i k}=(G B)_{n k}$ $-(G B)_{n-1, k}$ and the result follows from the equivalence of the relations (5) and (6).

Lemma 13. The one-to-one correspondence $A \leftrightarrow P$ defined above between $\Gamma_{0}\left(l_{1}, l_{1}\right)$ and $\Gamma(|c|,|c|)$ is an isomorphism.

Proof. Let $A, B \in \Gamma_{0}\left(l_{1}, l_{1}\right)$ and let the matrices corresponding to them in $\Gamma_{0}\left(l_{1},|c|\right), \Gamma(|c|,|c|)$ be $G, H$ and $P, Q$ respectively; that is, $A \leftrightarrow G \leftrightarrow P, B \leftrightarrow H \leftrightarrow Q$ and $G, H \in \Gamma_{0}\left(l_{1},|c|\right) ; P, Q \in \Gamma(|c|,|c|)$.

It is obvious that $A+B \leftrightarrow P+Q \in \Gamma(|c|,|c|), \lambda A \leftrightarrow \lambda P$ where $\lambda$ is any complex constant. [By Lemma 9 we have that $A+B, \lambda A$ and $A B$ all belong to $\Gamma_{0}\left(l_{1}, l_{1}\right)$.] We have to prove that $P Q \in \Gamma(|c|,|c|)$ and that $A B \leftrightarrow P Q$.

In view of Lemmas 10,11 and 12 it is enough to prove that the product $P Q$ exists and that

$$
(P Q)_{n k}=(G B)_{n k}-(G B)_{n, k+1} \quad(n, k=0,1,2, \cdots) .
$$

Now,

$$
\begin{aligned}
(G B)_{n k}-(G B)_{n, k+1} & =\sum_{i=0}^{\infty} g_{n i}\left(b_{i k}-b_{i, k+1}\right) \\
& =\sum_{i=0}^{\infty} g_{n i}\left(h_{i k}-h_{i-1, k}-h_{i, k+1}+h_{i-1, k+1}\right) \text { by }(6), \\
& =\sum_{i=0}^{\infty} g_{n i}\left\{\left(h_{i k}-h_{i, k+1}\right)+\left(h_{i-1, k+1}-h_{i-1, k}\right)\right\} \\
& =\sum_{i=0}^{\infty}\left(g_{n i}-g_{n, i+1}\right)\left(h_{i k}-h_{i, k-1}\right) \\
& =\sum_{i=0}^{\infty} p_{n i} q_{i k} .
\end{aligned}
$$

This establishes that the product $P Q$ exists and that it satisfies the relation (10). Since $A B \leftrightarrow G B \in \Gamma_{0}\left(l_{1},|c|\right)$ and $G B \leftrightarrow P Q \in \Gamma(|c|,|c|)$, the lemma is proved. 
Corollary. The sets $\Gamma(|c|,|c|)$ and $\Gamma\left(c_{0}, c_{0}\right)$ are isomorphic under the correspondence $P \leftrightarrow A^{\prime}$ where $A^{\prime}$ is the transpose of $A$.

THEOREM 3. The set $\Gamma(|c|,|c|)$ is a complex Banach algebra where the norm of $P \in \Gamma(|c|,|c|)$ is defined by $\|P\| *$ given in (3).

Proof. We have from the relation (3), $\|P\|^{*}=\|A\|^{\prime}=\left\|A^{\prime}\right\|$. Also, it is easily verified that the equation (3) defines a norm over $\Gamma(|c|,|c|)$. The theorem follows then from Lemma 2 and the corollary above, in view of the isometry.

Theorem 3 above leads us to the following generalization of Bosanquet's analogue [3], for absolute summability of Mercer's theorem; it is the analogue, for absolute summability, of Theorem 2.

THEOREM 4. If $P \in \Gamma(|c|,|c|)$ is a lower-semimatrix and

$$
\left|p_{n n}\right|-\sum_{k=n+1}^{\infty}\left|\sum_{i=n}^{k}\left(p_{k i}-p_{k-1, i}\right)\right|>\lambda>0
$$

for all $n=0,1,2, \cdots$, then $P x \in(|c|)$ implies $x \in(|c|)$.

Proof. Let $B=A^{\prime} \in \Gamma\left(c_{0}, c_{0}\right)$ correspond to $P$ under the isomorphism stated in the corollary to Lemma 13 . Then it is easily verified that $B$ satisfies the condition $\left|b_{n n}\right|-\sum_{k=n+1}^{\infty}\left|b_{n k}\right|>\lambda>0$ $(n=0,1,2, \cdots)$ and hence, as seen in the proof of Theorem 2, we have that $B^{-1} \in \Gamma\left(c_{0}, c_{0}\right)$. It follows now from the isomorphism that $P^{-1} \in \Gamma(|c|,|c|)$. Also, $P$ and $P^{-1}$ are lower-semimatrices and therefore if $P x \in(|c|)$ then $x=\left(P^{-1} P\right) x=P^{-1}(P x) \in(|c|)$ and the theorem is proved.

It is easily seen that the Mercerian theorem for absolute summability given by Love [ 8 , Theorem 5$]$ is an immediate corollary of Theorem 4 proved above. It can also be proved that the following result for Hausdorff matrices, which includes and is more general than the results given by Love [8, Corollaries 4,5$]$, is also a corollary of our Theorem 4 . We give an alternative short and interesting proof of the result.

Theorem 5. If $A \in \Gamma(c, c)$ is a Hausdorff matrix and satisfies the condition

$$
\left|a_{n n}\right|-\sum_{k=0}^{n-1}\left|a_{n k}\right|>\lambda>0 \quad(n=0,1,2, \cdots)
$$

then $A x \in(|c|)$ implies that $x \in(|c|)$.

Proof. As shown in the proof of Theorem 2, we have now 
$A^{-1} \in \Gamma(c, c)$, and since $A$ is Hausdorff, so is $A^{-1}$. Now, Knopp and Lorentz [6, Theorem 3] have proved that any Hausdorff matrix belonging to $\Gamma(c, c)$ belongs also to $\Gamma(|c|,|c|)$. Thus $A^{-1} \in \Gamma(|c|,|c|)$ and the theorem is immediately proved.

\section{REFERENCES}

1. R. P. Agnew, Mercer's summability theorem, J. London Math. Soc. vol. 29 (1954) pp. 123-125.

2. - Equivalence of methods for evaluation of sequences, Proc. Amer. Math. Soc. vol. 3 (1952) pp. 550-556.

3. L. S. Bosanquet, An analogue of Mercer's theorem, J. London Math. Soc. vol. 13 (1938) pp. 177-180.

4. G. H. Hardy, Divergent series, Oxford, 1949.

5. E. Hille, Functional analysis and semigroups, New York, 1948.

6. K. Knopp and G. Lorentz, Beitrage zur absoluten Limitierung, Archiv der Mathematik vol. 2 (1949-1950) pp. 10-16.

7. R. T. Leslie and E. R. Love, An extension of Mercer's theorem, Proc. Amer. Math. Soc. vol. 3 (1952) pp. 448-457.

8. E. R. Love, Mercer's summability theorem, J. London Math. Soc. vol. 27 (1952) pp. 413-429.

9. F. M. Mears, Absolute regularity and the Nörlund mean, Ann. of Math. (2) vol. 38 (1937) pp. 594-601.

10. J. Mercer, On the limits of real variants, Proc. London Math. Soc. (2) vol. 5 (1907) pp. 206-224.

11. M. R. Parameswaran, Some converse theorems on summability, Proceedings of the Indian Academy of Sciences (Sec. A) vol. 36 (1952) pp. 363-369.

12. - On the reciprocal of a K-matrix, J. Indian Math. Soc. vol. 20 (1956) pp. 329-331.

13. F. V. Sirkov, O teoreme Mercera, Uspehi Matematičeskih Nauk. (NS) vol. 10 (1955) pp. 167-170.

14. K. Zeller, Allgemeine Eigenschaften von Limitierungsverfahren, Math. Zeit. vol. 53 (1951) pp. 463-487.

Ramanujan Institute of Mathematics, Madras 